Article

\title{
Some Convolution Formulae Related to the Second-Order Linear Recurrence Sequence
}

\author{
Zhuoyu Chen ${ }^{1}$ and Lan Qi ${ }^{2, *(D)}$ \\ 1 School of Mathematics, Northwest University, Xi'an 710127, China; chenzymath@stumail.nwu.edu.cn \\ 2 School of Mathematics and Statistics, Yulin University, Yulin 719000, China \\ * Correspondence: qilanmail@163.com
}

Received: 9 May 2019; Accepted: 12 June 2019; Published: 13 June 2019

check for updates

\begin{abstract}
The main aim of this paper is that for any second-order linear recurrence sequence, the generating function of which is $f(t)=\frac{1}{1+a t+b t^{2}}$, we can give the exact coefficient expression of the power series expansion of $f^{x}(t)$ for $x \in \mathbf{R}$ with elementary methods and symmetry properties. On the other hand, if we take some special values for $a$ and $b$, not only can we obtain the convolution formula of some important polynomials, but also we can establish the relationship between polynomials and themselves. For example, we can find relationship between the Chebyshev polynomials and Legendre polynomials.
\end{abstract}

Keywords: Fibonacci numbers; Lucas numbers; Chebyshev polynomials; Legendre polynomials; Jacobi polynomials; Gegenbauer polynomials; convolution formula

MSC: $11 B 83$

\section{Introduction}

For any integer $n \geq 1$ and any real number $y$, the Fibonacci polynomials $F_{n}(y)$ and the Lucas polynomials $L_{n}(y)$ are defined by the second-order linear recurrence sequence

$$
F_{n+1}(y)=y F_{n}(y)+F_{n-1}(y)
$$

and

$$
L_{n+1}(y)=y L_{n}(y)+L_{n-1}(y),
$$

where the first two terms are $F_{0}(y)=0, F_{1}(y)=1, L_{0}(y)=2$ and $L_{1}(y)=y$.

If we take $\alpha=\frac{y+\sqrt{y^{2}+4}}{2}, \beta=\frac{y-\sqrt{y^{2}+4}}{2}$, according to the properties of the second-order linear recurrence sequence, we have

$$
F_{n}(y)=\frac{\alpha^{n}-\beta^{n}}{\alpha-\beta}
$$

and

$$
L_{n}(y)=\alpha^{n}+\beta^{n} .
$$

For any integer $n \geq 0$, the Fibonacci numbers $F_{n}=F_{n}(1)$ can be defined by the generating function

$$
\frac{1}{1-t-t^{2}}=\sum_{n=0}^{\infty} F_{n} t^{n} \text {. }
$$


For any integer $n \geq 0$, the first and the second kind Chebyshev polynomials $T_{n}(y)$ and $U_{n}(y)$ are defined by the second-order linear recurrence sequence

$$
T_{n+2}(y)=2 y T_{n+1}(y)-T_{n}(y)
$$

and

$$
U_{n+2}(y)=2 y U_{n+1}(y)-U_{n}(y),
$$

where the first two terms are $T_{0}(y)=1, T_{1}(y)=y, U_{0}(y)=1$ and $U_{1}(y)=2 y$.

If we take $\alpha=y+\sqrt{y^{2}-1}, \beta=y-\sqrt{y^{2}-1}$, according to the properties of the second-order linear recurrence sequence, we have

$$
T_{n}(y)=\frac{\alpha^{n}+\beta^{n}}{2}
$$

and

$$
U_{n}(y)=\frac{\alpha^{n+1}-\beta^{n+1}}{\alpha-\beta} .
$$

On the other hand, the second kind Chebyshev polynomials $U_{n}(y)$ can be also defined by the generating function

$$
\frac{1}{1-2 y t+t^{2}}=\sum_{n=0}^{\infty} U_{n}(y) t^{n}
$$

Besides Fibonacci polynomials, Lucas polynomials and Chebyshev polynomials, other orthogonal polynomials have also been studied by interested scholars.

For example, the Legendre polynomials $P_{n}(y)$ are defined by the generating function

$$
\left(\frac{1}{1-2 y t+t^{2}}\right)^{\frac{1}{2}}=\sum_{n=0}^{\infty} P_{n}(y) t^{n} .
$$

The Jacobi polynomials $\left\{P_{n}^{(\alpha, \beta)}(y)\right\}_{0 \leq n<\infty}$ are defined by the generating function

$$
\left[R(1+R-t)^{\alpha}(1+R+t)^{\beta}\right]^{-1}=\sum_{k=0}^{\infty} 2^{-\alpha-\beta} P_{n}^{(\alpha, \beta)}(y) t^{n},
$$

where $R=\sqrt{1-2 y t+t^{2}},|t|<1, \alpha, \beta>-1$.

The Gegenbauer polynomials $\left\{C_{n}^{\lambda}(y)\right\}_{0 \leq n<\infty}$ are defined by the generating function

$$
\left(\frac{1}{1-2 y t+t^{2}}\right)^{\lambda}=\sum_{n=0}^{\infty} C_{n}^{\lambda}(y) t^{n},\left(\lambda>-\frac{1}{2}\right)
$$

It is well know that polynomials and sequence occupy indispensable positions in the research of number theory. Especially, Fibonacci and Lucas numbers, Chebyshev and Legendre polynomials and others. These polynomials and numbers are closely related and there are a variety of meaningful results which have been researched by interested scholars until now. For example, the identities of Chebyshev polynomials can be found in [1-9], and the contents about Fibonacci and Lucas numbers in $[10,11]$. Some authors have a research which connects Chebyshev polynomials and Fibonacci or Lucas polynomials (see [12-14]).

In particular, we can find many significant results in the aspect of studying the calculating problem of one kind sums of some important polynomials. For example, Yuankui Ma and Wenpeng Zhang have calculated one kind sums of Fibonacci Polynomials (see [15]) as follows. 
Let $h$ be a positive integer, for any integer $n \geq 0$, they proved

$$
\begin{array}{r}
\sum_{a_{1}+a_{2}+\cdots+a_{h+1}=n} F_{a_{1}}(x) F_{a_{2}}(x) \cdots F_{a_{h+1}}(x)=\frac{1}{h !} \cdot \sum_{j=1}^{h} \frac{(-1)^{h-j} \cdot S(h, j)}{x^{2 h-j}} \\
\times\left(\sum_{i=0}^{n} \frac{(n-i+j) !}{(n-i) !} \cdot\left(\begin{array}{c}
2 h+i-j-1 \\
i
\end{array}\right) \cdot \frac{(-1)^{i} \cdot 2^{i} \cdot F_{n-i+j}(x)}{x^{i}}\right),
\end{array}
$$

where the summation is over all $h+1$-tuples with non-negative integer coordinates $\left(a_{1}, a_{2}, \cdots, a_{h+1}\right.$ such that $a_{1}+a_{2}+\cdots+a_{h+1}=n$, and $S(h, i)$ is a second order non-linear recurrence sequence defined by $S(h, 0)=0, S(h, h)=1$, and $S(h+1, i+1)=2 \cdot(2 h-1-i) \cdot S(h, i+1)+S(h, i)$ for all positive integers $1 \leq i \leq h-1$.

Yixue Zhang and Zhuoyu Chen have researched the calculating problem of one kind sums of the second kind Chebyshev polynomials (see [16]) as follows.

Let $h$ be a positive integer, for any integer $n \geq 0$, they proved

$$
\begin{aligned}
& \sum_{a_{1}+a_{2}+\cdots+a_{h+1}=n} U_{a_{1}}(x) U_{a_{2}}(x) \cdots U_{a_{h+1}}(x) \\
= & \frac{1}{2^{h} \cdot h !} \cdot \sum_{j=1}^{h} \frac{C(h, j)}{x^{2 h-j}} \sum_{i=0}^{n} \frac{(n-i+j) !}{(n-i) !} \cdot\left(\begin{array}{c}
2 h+i-j-1 \\
i
\end{array}\right) \cdot \frac{U_{n-i+j}(x)}{x^{i}},
\end{aligned}
$$

where $C(h, i)$ is a second order non-linear recurrence sequence defined by $C(h, 0)=0, C(h, h)=1$, $C(h+1,1)=1 \cdot 3 \cdot 5 \cdots(2 h-1)=(2 h-1) ! !$ and $C(h+1, i+1)=(2 h-1-i) \cdot C(h, i+1)+C(h, i)$ for all $1 \leq i \leq h-1$.

Shimeng Shen and Li Chen have studied the calculating problem of one kind sums of Legendre Polynomials (see [17]) as follows.

For any positive integer $k$ and integer $n \geq 0$, they proved

$$
\begin{aligned}
& (2 k-1) ! ! \sum_{a_{1}+a_{2}+\cdots+a_{2 k+1}=n} P_{a_{1}}(x) P_{a_{2}}(x) \cdots P_{a_{k}}(x) \\
= & \sum_{j=1}^{k} C(k, j) \sum_{i=0}^{n} \frac{(n+k+1-i-j) !}{(n-i) !} \cdot \frac{\left(\begin{array}{l}
i+j+k-2 \\
i
\end{array}\right)}{x^{k-1+i+j}} \cdot P_{n+k+1-i-j}(x)
\end{aligned}
$$

where $(2 k-1) ! !=1 \times 3 \times 5 \cdots(2 k-1)=2^{k}\left(\frac{1}{2}\right)_{k}$, and $C(k, i)$ is a recurrence sequence defined by $C(k, 1)=1, C(k+1, k+1)=(2 k-1) ! !$ and $C(k+1, i+1)=C(k, i+1)+(k-1+i) \cdot C(k, i)$ for all $1 \leq i \leq k-1$.

They have converted the complex sums of $F_{n}(x)$ into a simple combination of $F_{n}(x)$, the complex sums of $U_{n}(x)$ into a simple combination of $U_{n}(x)$, and the complex sums of $P_{n}(x)$ into a simple combination of $P_{n}(x)$.

Very recently, Taekyun Kim and other people researched the properties of Fibonacci numbers through introducing the convolved Fibonacci numbers $p_{n}(x)$ by generating function as follows (see [18]):

$$
\left(\frac{1}{1-t-t^{2}}\right)^{x}=\sum_{n=0}^{\infty} p_{n}(x) \frac{t^{n}}{n !},(x \in \mathbf{R})
$$

They researched some new and explicit identities of the convolved Fibonacci numbers for $x \in \mathbf{N}$. For example, for $n \geq 0$ and $r \in \mathbf{N}$, they have proved the recurrence relationship of $p_{n}(x)$ (see [18]):

$$
p_{n}(x)=\sum_{l=0}^{n} p_{l}(r) p_{n-l}(x-r)=\sum_{l=0}^{n} p_{n-l}(r) p_{l}(x-r) .
$$


The convolved Fibonacci numbers $p_{n}(x)$ seems to be only connected with the simple power square. In fact, it can establish the relationship between polynomials and themselves, so the further research of $p_{n}(x)$ is very significant. They have provided us a new perspective to study the properties of some vital polynomials. For example, Taekyun Kim and other people have proved the relationship between $p_{n}(x)$ and the combination sums about Fibonacci numbers:

$$
\frac{p_{n}(r+1)}{n !}=\sum_{l_{1}=0}^{n} \sum_{l_{2}=0}^{n-l_{1}} \cdots \sum_{l_{r}=0}^{n-l_{1}-\cdots-l_{r-1}} F_{l_{1}} F_{l_{2}} \cdots F_{l_{r}} F_{n-l_{1}-l_{2}-\cdots-l_{r}} .
$$

They have converted the complex sums of $F_{n}(x)$ into a calculation problem of $p_{n}(x)$ and the calculation method is easier and the expression is simpler.

Inspired by this article, in this paper, for any second-order linear recurrence sequence, the generating function of which is $f(t)=\frac{1}{1+a t+b t^{2}}$, we can define

$$
\left(\frac{1}{1+a t+b t^{2}}\right)^{x}=\sum_{n=0}^{\infty} p_{n}(x) \frac{t^{n}}{n !},(a, b, x \in \mathbf{R})
$$

Firstly, we give a specific computational formula of $p_{n}(x)$ for $x \in \mathbf{R}$ using the elementary methods. After that for any polynomial or sequence, the generating function of which is $f(t)=\frac{1}{1+a t+b t^{2}}$, we can obtain its convolved formula easily and directly.

Secondly, if we take some special values for $a, b$ in $f(t)$ and $x$ in $p_{n}(x)$, we can find some relationship between special polynomials and themselves. For example, we will establish the relationship between the convolved Fibonacci numbers and Lucas numbers, the relationship between the convolved formula of the second kind Chebyshev polynomials and the first kind Chebyshev polynomials, and the relationship between Legendre polynomials and the first kind Chebyshev polynomials and others.

At last, through the computational formula of $p_{n}(x)$, especially for $x \in \mathbf{N}$, we can also convert the complex sums of $F_{n}$ into a liner combination of $L_{n}$; and express the complex sums of $U_{n}(y)$ as a liner combination of $T_{n}(y)$. More importantly, the forms are more common and the calculations are easier than previous results.

We will prove the main results as follows:

Theorem 1. Let $f(t)=\frac{1}{1-t-t^{2}}$, for any integer $n \geq 0$ and $x \in \mathbf{R}$, we can obtain

$$
p_{n}(x)=\frac{1}{2} \sum_{i=0}^{n}(-1)^{i}\left(\begin{array}{c}
n \\
i
\end{array}\right)\langle x\rangle_{i}\langle x\rangle_{n-i} L_{n-2 i}
$$

where $\langle x\rangle_{n}=x(x+1)(x+2) \cdots(x+n-1)$ and $(x)_{0}=1$.

Theorem 2. Let $f(t)=\frac{1}{1-2 y t+t^{2}}$, for any integer $n \geq 0$ and $x, y \in \mathbf{R}$, we can obtain

$$
p_{n}(x ; y)=\sum_{i=0}^{n}\left(\begin{array}{c}
n \\
i
\end{array}\right)\langle x\rangle_{i}\langle x\rangle_{n-i} T_{n-2 i}(y)
$$

From Theorem 1 we can deduce the following: 
Corollary 1. For any positive integer $k$, we have the identity

$$
\begin{aligned}
& \sum_{a_{1}+a_{2}+\cdots+a_{k}=n} F_{a_{1}} F_{a_{2}} \cdots F_{a_{k}} \\
= & \frac{1}{2((k-1) !)^{2}} \sum_{i=0}^{n}(-1)^{i} \frac{(k+i-1) !(k+n-i-1) !}{i !(n-i) !} \cdot L_{n-2 i} .
\end{aligned}
$$

From Theorem 2 we can deduce the following:

Corollary 2. For any positive integer $k$, we have the identity

$$
\begin{aligned}
& \sum_{a_{1}+a_{2}+\cdots+a_{k}=n} U_{a_{1}}(y) \cdot U_{a_{2}}(y) \cdots U_{a_{k}}(y) \\
= & \frac{1}{((k-1) !)^{2}} \sum_{i=0}^{n} \frac{(k+i-1) !(k+n-i-1) !}{i !(n-i) !} \cdot T_{n-2 i}(y) .
\end{aligned}
$$

Corollary 3. If $x=\frac{1}{2}$, we have the identity

$$
P_{n}(y)=\frac{1}{2^{n}} \sum_{i=0}^{n} \frac{(2 i-1) ! !(2 n-2 i-1) ! !}{i !(n-i) !} \cdot T_{n-2 i}(y) .
$$

Corollary 4. If $x=-\frac{1}{2}$, we have the identity

$$
R=\sum_{n=0}^{\infty} \frac{1}{2^{n}} \sum_{i=0}^{n} \frac{(2 i-3) ! !(2 n-2 i-3) ! !}{i !(n-i) !} \cdot T_{n-2 i}(y) \cdot t^{n} .
$$

Corollary 5. If $x=\lambda>-\frac{1}{2}$, we have the identity

$$
C_{n}^{\lambda}(y)=\frac{1}{n !} \sum_{i=0}^{n}\left(\begin{array}{c}
n \\
i
\end{array}\right)\langle\lambda\rangle_{i}\langle\lambda\rangle_{n-i} T_{n-2 i}(y) .
$$

Theorems 1 and 2 give the computational formula of $p_{n}(x)$ of some famous polynomials. Especially, we know that polynomials are closely connected and they can be converted to each other. According to these theorems, we can obtain the relationship between the polynomials easily. It cannot only extend the application of orthogonal polynomials, but also make replacement calculations according to its complexity. For example, if we make a calculation involving the Gegenbauer polynomials, for simple calculations, we can convert it into Chebyshev polynomials according to Corollary 5.

\section{A Simple Lemma}

In order to prove our theorems, we are going to introduce a simple lemma.

Lemma 1. For any integer $n \geq 0$ and $a, b, x \in \mathbf{R}$, we can obtain the equation

$$
p_{n}(x)=\frac{1}{2} \sum_{i=0}^{n} b^{i}\left(\begin{array}{c}
n \\
i
\end{array}\right)\langle x\rangle_{i}\langle x\rangle_{n-i}\left(\left(\frac{-a+\sqrt{a^{2}-4 b}}{2}\right)^{n-2 i}+\left(\frac{-a-\sqrt{a^{2}-4 b}}{2}\right)^{n-2 i}\right) .
$$

Proof. Firstly, according Equation (1), we have

$$
\sum_{n=0}^{\infty} p_{n}(x) \frac{t^{n}}{n !}=\left(\frac{1}{1+a t+b t^{2}}\right)^{x}=(1-\alpha t)^{-x}(1-\beta t)^{-x} .
$$


We can easily know that $\alpha+\beta=-a, \alpha \beta=b$ and $\alpha=\frac{-a+\sqrt{a^{2}-4 b}}{2}, \beta=\frac{-a-\sqrt{a^{2}-4 b}}{2}$ are two roots of $1+a t+b t^{2}=0$.

Then, applying the properties of power series, we obtain

$$
(1-\alpha t)^{-x}=\sum_{n=0}^{\infty}\left(\begin{array}{c}
-x \\
n
\end{array}\right)(-1)^{n}(\alpha t)^{n}=\sum_{n=0}^{\infty} \frac{(-x)_{n}}{n !}(-1)^{n} \alpha^{n} t^{n}
$$

and

$$
(1-\beta t)^{-x}=\sum_{n=0}^{\infty}\left(\begin{array}{c}
-x \\
n
\end{array}\right)(-1)^{n}(\beta t)^{n}=\sum_{n=0}^{\infty} \frac{(-x)_{n}}{n !}(-1)^{n} \beta^{n} t^{n}
$$

where $(x)_{n}=x(x-1)(x-2) \cdots(x-n+1)$ and $(x)_{0}=1$.

Combining Equations (2)-(4), we get

$$
\begin{aligned}
\sum_{n=0}^{\infty} p_{n}(x) \frac{t^{n}}{n !} & =\left(\sum_{n=0}^{\infty} \frac{(-x)_{n}}{n !}(-1)^{n} \alpha^{n} t^{n}\right)\left(\sum_{n=0}^{\infty} \frac{(-x)_{n}}{n !}(-1)^{n} \beta^{n} t^{n}\right) \\
& =\sum_{n=0}^{\infty}\left(\sum_{i=0}^{n} \frac{(-x)_{i}(-1)^{i} \alpha^{i} t^{i}}{i !} \cdot \frac{(-x)_{n-i}(-1)^{n-i} \beta^{n-i} t^{n-i}}{(n-i) !}\right) \\
& =\sum_{n=0}^{\infty} \frac{(-1)^{n}}{n !}\left(\sum_{i=0}^{n}\left(\begin{array}{c}
n \\
i
\end{array}\right)(-x)_{i}(-x)_{n-i} \alpha^{i} \beta^{n-i}\right) t^{n} .
\end{aligned}
$$

Similarly, according the symmetry of $\alpha$ and $\beta$, we can easily obtain

$$
\sum_{n=0}^{\infty} p_{n}(x) \frac{t^{n}}{n !}=\sum_{n=0}^{\infty} \frac{(-1)^{n}}{n !}\left(\sum_{i=0}^{n}\left(\begin{array}{l}
n \\
i
\end{array}\right)(-x)_{i}(-x)_{n-i} \beta^{i} \alpha^{n-i}\right) t^{n}
$$

Then, combining Equations (5) and (6), we know that

$$
\begin{aligned}
\sum_{n=0}^{\infty} p_{n}(x) \frac{t^{n}}{n !} & =\frac{1}{2} \sum_{n=0}^{\infty} \frac{(-1)^{n}}{n !}\left(\sum_{i=0}^{n}\left(\begin{array}{l}
n \\
i
\end{array}\right)(-x)_{i}(-x)_{n-i}(\alpha \beta)^{i}\left(\beta^{n-2 i}+\alpha^{n-2 i}\right)\right) t^{n} \\
& =\frac{1}{2} \sum_{n=0}^{\infty} \frac{1}{n !}\left(\sum_{i=0}^{n} b^{i}\left(\begin{array}{l}
n \\
i
\end{array}\right)\langle x\rangle_{i}\langle x\rangle_{n-i}\left(\beta^{n-2 i}+\alpha^{n-2 i}\right)\right) t^{n} .
\end{aligned}
$$

Comparing the coefficients of $t^{n}$ in Equation (7), we get

$$
\begin{aligned}
p_{n}(x) & =\frac{1}{2} \sum_{i=0}^{n} b^{i}\left(\begin{array}{l}
n \\
i
\end{array}\right)\langle x\rangle_{i}\langle x\rangle_{n-i}\left(\alpha^{n-2 i}+\beta^{n-2 i}\right) \\
& =\frac{1}{2} \sum_{i=0}^{n} b^{i}\left(\begin{array}{l}
n \\
i
\end{array}\right)\langle x\rangle_{i}\langle x\rangle_{n-i}\left(\left(\frac{-a+\sqrt{a^{2}-4 b}}{2}\right)^{n-2 i}+\left(\frac{-a-\sqrt{a^{2}-4 b}}{2}\right)^{n-2 i}\right) .
\end{aligned}
$$

Now we have completed the proof of the Lemma 1.

\section{Proof of the Theorem}

Proof of Theorem 1. If we take $a=-1$ and $b=-1$ in Equation (1), we know that $f(t)$ is the generating function of Fibonacci number. That is,

$$
f(t)=\frac{1}{1-t-t^{2}}=\sum_{n=0}^{\infty} F_{n} t^{n}
$$


The convolved Fibonacci numbers $p_{n}(x)$ are defined by the generating function as [18]

$$
f^{x}(t)=\left(\frac{1}{1-t-t^{2}}\right)^{x}=\sum_{n=0}^{\infty} p_{n}(x) \frac{t^{n}}{n !} .
$$

In this time, $\alpha=\frac{1+\sqrt{5}}{2}, \beta=\frac{1-\sqrt{5}}{2}$.

According to the Lemma 1 and $L_{n}=\alpha^{n}+\beta^{n}$, we can get

$$
\begin{aligned}
p_{n}(x) & =\frac{1}{2} \sum_{i=0}^{n} b^{i}\left(\begin{array}{c}
n \\
i
\end{array}\right)\langle x\rangle_{i}\langle x\rangle_{n-i}\left(\left(\frac{-a+\sqrt{a^{2}-4 b}}{2}\right)^{n-2 i}+\left(\frac{-a-\sqrt{a^{2}-4 b}}{2}\right)^{n-2 i}\right) \\
& =\frac{1}{2} \sum_{i=0}^{n} b^{i}\left(\begin{array}{c}
n \\
i
\end{array}\right)\langle x\rangle_{i}\langle x\rangle_{n-i}\left(\left(\frac{1+\sqrt{5}}{2}\right)^{n-2 i}+\left(\frac{1-\sqrt{5}}{2}\right)^{n-2 i}\right) \\
& =\frac{1}{2} \sum_{i=0}^{n} b^{i}\left(\begin{array}{c}
n \\
i
\end{array}\right)\langle x\rangle_{i}\langle x\rangle_{n-i}\left(\alpha^{n-2 i}+\beta^{n-2 i}\right) \\
& =\frac{1}{2} \sum_{i=0}^{n}(-1)^{i}\left(\begin{array}{c}
n \\
i
\end{array}\right)\langle x\rangle_{i}\langle x\rangle_{n-i} L_{n-2 i} .
\end{aligned}
$$

In this equation, $p_{n}(x)$ is expressed as a combined forms of Lucas number. The Proof of Theorem 1 has finished.

About the convolved Fibonacci numbers $p_{n}(x)$, Taekyun Kim and others have obtained its some-recurrence formulae in reference [18]. Based on [18], we have given an exact computational formula of $p_{n}(x)$ for any arbitrary $x$ in Theorem 1. Compared with the results in [18], Theorem 1 is more general and easier.

If we take $x=k \in \mathbf{N}$ in Equation (8), we get

$$
\begin{aligned}
& \sum_{n=0}^{\infty} p_{n}(k) \frac{t^{n}}{n !}=\left(\frac{1}{1-t-t^{2}}\right)^{k}=\left(\sum_{n=0}^{\infty} F_{n} t^{n}\right)^{k} \\
= & \left(\sum_{a_{1}=0}^{\infty} F_{a_{1}} \cdot t^{a_{1}}\right)\left(\sum_{a_{2}=0}^{\infty} F_{a_{2}} \cdot t^{a_{2}}\right) \cdots\left(\sum_{a_{k}=0}^{\infty} F_{a_{k}} \cdot t^{a_{k}}\right) \\
= & \left(\sum_{a_{1}=0}^{\infty} \sum_{a_{2}=0}^{\infty} \cdots \sum_{a_{k}=0}^{\infty} F_{a_{1}} \cdot F_{a_{2}} \cdots F_{a_{k}} \cdot t^{a_{1}+a_{2} \cdots+a_{k}}\right) \\
= & \sum_{n=0}^{\infty}\left(\sum_{a_{1}+a_{2}+\cdots+a_{k}=n} F_{a_{1}} \cdot F_{a_{2}} \cdots F_{a_{k}}\right) \cdot t^{n},
\end{aligned}
$$

and then combining Equation (9), we can obtain

$$
\begin{aligned}
& \sum_{a_{1}+a_{2}+\cdots+a_{k}=n} F_{a_{1}} \cdot F_{a_{2}} \cdots F_{a_{k}} \\
= & \frac{1}{2 n !} \sum_{i=0}^{n}(-1)^{i}\left(\begin{array}{c}
n \\
i
\end{array}\right)\langle k\rangle_{i}\langle k\rangle_{n-i} L_{n-2 i} \\
= & \frac{1}{2((k-1) !)^{2}} \sum_{i=0}^{n}(-1)^{i} \frac{(k+i-1) !(k+n-i-1) !}{i !(n-i) !} L_{n-2 i} .
\end{aligned}
$$

The proof of Corollary 1 has finished.

For every $F_{a_{l}}(1 \leq l \leq k), \sum_{a_{1}+a_{2}+\cdots+a_{k}=n} F_{a_{1}} \cdot F_{a_{2}} \cdots F_{a_{k}}$ is symmetry. 
Proof of Theorem 2. If we take $a=-2 y$ and $b=1$ in Equation (1), we all know $f(t ; y)$ is the generating function of the second-kind Chebyshev polynomials $U_{n}(y)$

$$
f(t ; y)=\frac{1}{1-2 y t+t^{2}}=\sum_{n=0}^{\infty} U_{n}(y) t^{n}
$$

The convolved second-kind Chebyshev polynomials $p_{n}(x ; y)$ are defined by the generating function as [18]

$$
f^{x}(t ; y)=\left(\frac{1}{1-2 y t+t^{2}}\right)^{x}=\sum_{n=0}^{\infty} p_{n}(x ; y) \frac{t^{n}}{n !} .
$$

In this time, $\alpha=y+\sqrt{y^{2}-1}, \beta=y-\sqrt{y^{2}-1}$.

According to the Lemma 1 and $T_{n}(y)=\frac{1}{2}\left(\alpha^{n}+\beta^{n}\right)$, we can get

$$
p_{n}(x ; y)=\sum_{i=0}^{n}\left(\begin{array}{c}
n \\
i
\end{array}\right)\langle x\rangle_{i}\langle x\rangle_{n-i} T_{n-2 i}(y)
$$

In this equation, $p_{n}(x ; y)$ is expressed as a combined form of the first-kind Chebyshev polynomials $T_{n}(x)$.

If we take $x=k \in \mathbf{N}$ in Equation (10), and combining Equation (11) we can easily prove the Corollary 2.

Take $x=\frac{1}{2}$ in Equation (10), we know $f^{\frac{1}{2}}(t ; y)$ is the generating function of the Legendre polynomials $P_{n}(x)$ as follows:

$$
f^{\frac{1}{2}}(t)=\left(\frac{1}{1-2 y t+t^{2}}\right)^{\frac{1}{2}}=\sum_{n=0}^{\infty} P_{n}(y) t^{n}=\sum_{n=0}^{\infty} p_{n}\left(\frac{1}{2} ; y\right) \frac{t^{n}}{n !} .
$$

According to Theorem 2, we can easily obtain

$$
\begin{aligned}
p_{n}\left(\frac{1}{2^{2}} ; y\right) & =\sum_{i=0}^{n}\left(\begin{array}{c}
n \\
i
\end{array}\right)\left\langle\frac{1}{2}\right\rangle_{i}\left\langle\frac{1}{2}\right\rangle_{n-i} T_{n-2 i}(y) \\
& =\frac{1}{2^{2 n}} \sum_{i=0}^{n} \frac{n !(2 i) !(2(n-i)) !}{(i !)^{2}((n-i) !)^{2}} \cdot T_{n-2 i}(y) \\
& =\frac{n !}{2^{n}} \sum_{i=0}^{n} \frac{(2 i-1) ! !(2 n-2 i-1) ! !}{i !(n-i) !} \cdot T_{n-2 i}(y) .
\end{aligned}
$$

In a word, we know the Legendre polynomials $P_{n}(x)$ can be expressed as combined forms of the first kind Chebyshev polynomials $T_{n}(x)$ as follows:

$$
P_{n}(y)=\frac{1}{2^{n}} \sum_{i=0}^{n} \frac{(2 i-1) ! !(2 n-2 i-1) ! !}{i !(n-i) !} \cdot T_{n-2 i}(y) .
$$

The proof of Corollary 3 has finished.

If we take $x=-\frac{1}{2}$ in (10), then we can easily obtain

$$
\begin{aligned}
R & =\sum_{n=0}^{\infty} p\left(-\frac{1}{2} ; y\right) \frac{t^{n}}{n !}=\sum_{n=0}^{\infty} \frac{1}{2^{2 n-2}} \sum_{i=0}^{n} \frac{(2(i-1)) !(2(n-i-1)) !}{i !(i-1) !(n-i) !(n-i-1) !} \cdot T_{n-2 i}(y) \cdot t^{n} \\
& =\sum_{n=0}^{\infty} \frac{1}{2^{n}} \sum_{i=0}^{n} \frac{(2 i-3) ! !(2 n-2 i-3) ! !}{i !(n-i) !} \cdot T_{n-2 i}(y) \cdot t^{n} .
\end{aligned}
$$


The proof of Corollary 4 has finished.

Taking $x=\lambda>-\frac{1}{2}$ in Equation (10), we know $f^{\lambda}(t ; y)$ is the generating function of the Gegenbauer polynomials $\left\{C_{n}^{\lambda}(y)\right\}_{0 \leq n<\infty}$ as follows:

$$
\left(\frac{1}{1-2 y t+t^{2}}\right)^{\lambda}=\sum_{n=0}^{\infty} C_{n}^{\lambda}(y) t^{n}=f^{\lambda}(t ; y)=\sum_{n=0}^{\infty} p_{n}(\lambda ; y) \frac{t^{n}}{n !}
$$

According to Theorem 2, we can easily obtain

$$
p_{n}(\lambda ; y)=\sum_{i=0}^{n}\left(\begin{array}{c}
n \\
i
\end{array}\right)\langle\lambda\rangle_{i}\langle\lambda\rangle_{n-i} T_{n-2 i}(y)
$$

The proof of Corollary 5 has finished.

Author Contributions: Writing—original draft: Z.C.; Writing—review and editing: L.Q.

Funding: This work is supported by the N. S. F. (11771351), (11826205), (11826203) of P. R. China and N.S.B.R.P in Shaanxi Province (2018JQ1093).

Acknowledgments: The authors would like to thank the referees for their very helpful and detailed comments, which have significantly improved the presentation of this paper.

Conflicts of Interest: The authors declare no conflict of interest.

\section{References}

1. Chen, L.; Zhang, W. Chebyshev polynomials and their some interesting applications. Adv. Differ. Equ. 2017, 2017, 303.

2. Wang, S. Some new identities of Chebyshev polynomials and their applications. Adv. Differ. Equ. 2015, 2015, 335.

3. Li, X. Some identities involving Chebyshev polynomials. Math. Probl. Eng. 2015, 2015, 950695. [CrossRef]

4. Ma, Y.; Lv, X. Several identities involving the reciprocal sums of Chebyshev polynomials. Math. Probl. Eng. 2017, 2017, 4194579. [CrossRef]

5. Cesarano, C. Identities and generating functions on Chebyshev polynomials. Georgian Math. J. 2012, 19, 427-440. [CrossRef]

6. Cesarano, C. Integral representations and new generating functions of Chebyshev polynomials. Hacet. J. Math. Stat. 2015, 44, 535-546. [CrossRef]

7. Lee, C.; Wong, K. On Chebyshev's Polynomials and Certain Combinatorial Identities. Bull. Malays. Math. Sci. Soc. 2011, 34, 279-286.

8. Wang, T.; Zhang, H. Some identities involving the derivative of the first kind Chebyshev polynomials. Math. Probl. Eng. 2015, 2015, 146313. [CrossRef]

9. Wang, T.; Zhang, W. Two identities involving the integral of the first kind Chebyshev polynomials. Bull. Math. Soc. Sci. Math. Roum. 2017, 108, 91-98.

10. Zhang, W. Some identities involving the Fibonacci numbers and Lucas numbers. Fibonacci Q. 2004, $42,149-154$.

11. Ma, R.; Zhang, W. Several identities involving the Fibonacci numbers and Lucas numbers. Fibonacci Q. 2007, $45,164-170$.

12. Kim, T.; Kim, D.; Dolgy, D.; Park, J. Sums of finite products of Chebyshev polynomials of the second kind and of Fibonacci polynomials. J. Inequal. Appl. 2018, 2018, 148. [CrossRef] [PubMed]

13. Kim, T.; Kim, D.; Kwon, J.; Dolgy, D. Expressing Sums of Finite Products of Chebyshev Polynomials of the Second Kind and of Fibonacci Polynomials by Several Orthogonal Polynomials. Mathematics 2018, 6, 210. [CrossRef]

14. Kim, T.; Kim, D.; Dolgy, D.; Kwon, J. Representing Sums of Finite Products of Chebyshev Polynomials of the First Kind and Lucas Polynomials by Chebyshev Polynomials. Mathematics 2019, 7, 26. [CrossRef]

15. Ma, Y.; Zhang, W. Some Identities Involving Fibonacci Polynomials and Fibonacci Numbers. Mathematics 2018, 6, 334. [CrossRef] 
16. Zhang, Y.; Chen, Z. A New Identity Involving the Chebyshev Polynomials. Mathematics 2018, 6, 244. [CrossRef]

17. Shen, S.; Chen, L. Some Types of Identities Involving the Legendre Polynomials. Mathematics 2019, 7, 114. [CrossRef]

18. Kim, T.; Dolgy, D.; Kim, D.; Seo, J. Convolved Fibonacci Numbers and Their Applications. ARS Comb. 2017, 135, 119-131.

(C) 2019 by the authors. Licensee MDPI, Basel, Switzerland. This article is an open access article distributed under the terms and conditions of the Creative Commons Attribution (CC BY) license (http://creativecommons.org/licenses/by/4.0/). 